\title{
A NEW LATTICE FORMULATION OF THE CONTINUUM
}

\author{
Yang Pang ${ }^{1,2}$ and Haicang Ren $^{3}$ \\ ${ }^{1}$ Columbia University, New York, NY 10027 \\ 2 Brookhaven National Laboratory, Upton, NY 11973 \\ ${ }^{3}$ Rockefeller University, New York, NY 10021
}

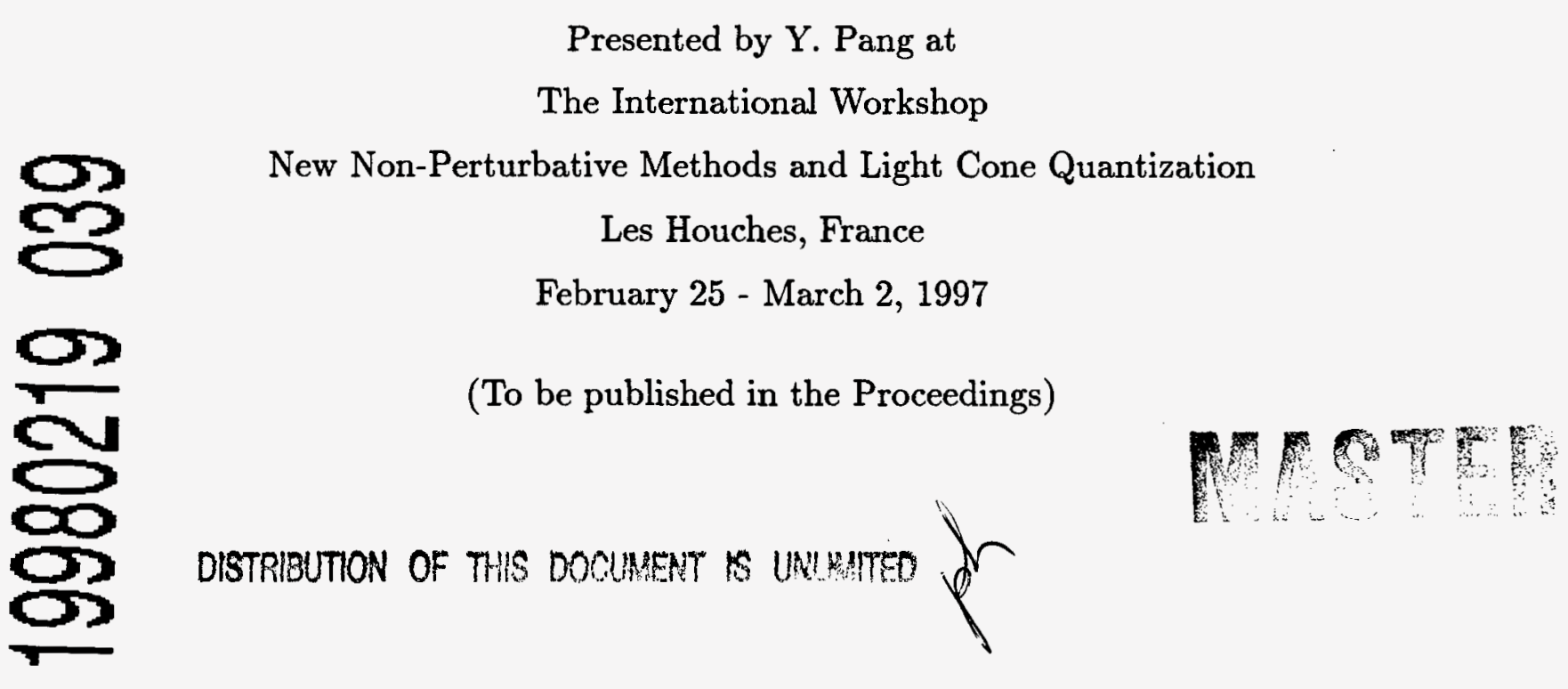

This manuscript has been authored under contract number DE-AC02-76CH00016 with the U.S. Department of Energy. Accordingly, the U.S. Government retains a non-exclusive, royalty-free license to publish or reproduce the published form of this contribution, or allow others to do so, for U.S. Government purposes. 


\section{DISCLAIMER}

This report was prepared as an account of work sponsored by an agency of the United States Government. Neither the United States Government nor any agency thereof, nor any of their employees, make any warranty, express or implied, or assumes any legal liability or responsibility for the accuracy, completeness, or usefulness of any information, apparatus, product, or process disclosed, or represents that its use would not infringe privately owned rights. Reference herein to any specific commercial product, process, or service by trade name, trademark, manufacturer, or otherwise does not necessarily constitute or imply its endorsement, recommendation, or favoring by the United States Government or any agency thereof. The views and opinions of authors expressed herein do not necessarily state or reflect those of the United States Government or any agency thereof. 
LECTURE 10

\title{
A New Lattice Formulation of the Continuum
}

\author{
Yang $\operatorname{Pang}\left({ }^{1,2}\right)$ and Haicang Ren $\left({ }^{3}\right)$ \\ (1) Department of Physics, Columbia University \\ New York, NY, 10027, USA \\ ( $\left.{ }^{2}\right)$ Department of Physics, Brookhaven National Laboratory \\ Upton, NY 11973, USA \\ $\left({ }^{3}\right)$ Department of Physics, Rockefeller University \\ New York, NY 10021, USA
}

$\mathbf{x}$

\section{INTRODUCTION}

It is well known that the Dirac equation on a discrete lattice in $D$ dimension has $2^{D}$ degenerate solutions. The usual method of removing these spurious solutions encounters difficulties with chiral symmetry when the lattice spacing $\ell \neq 0$, as demonstrated by the persistent problem of pion and kaon masses. On the other hand, we recall that in any crystal in nature, all the electrons do move in a lattice and satisfy the Dirac equation; yet there is not a single physical result that has ever been entangled with a spurious fermion solutions. Therefore it should not be difficult to eliminate these unphysical elements.

On a discrete lattice, particles hop from point to point, whereas in a real crystal the lattice structure is embedded in a continuum and electrons move continuously from lattice cell to lattice cell. In a discrete system, the lattice function are defined only on individual points (or links, as in the case of gauge fields). However, in a crystal the electron state vector is represented by the Bloch wave functions which are continuous functions in $\vec{r}$, and herein lies one of the essential differences. 
In this new approach [1][2] we shall expand the field operator in terms of suitably chosen complete set of orthonormal Bloch functions

$$
\left\{f_{n}(\vec{K} \mid \vec{r})\right\}
$$

where $\vec{K}$ denotes the Bloch wavenumber restricted to the Brillouin zone, and $n$ labels the different bands. Thus, $e^{-i \vec{K} \cdot \vec{r}} f_{n}(\vec{K} \mid \vec{r})$ has the periodicity of the lattice. The lattice approximation is then derived by either restricting it to only one band (say, $n=0$ ), or to a few appropriately defined low-lying bands. Since the inclusion of all band is the original continuum problem, there is a natural connection between the lattice and the continuum in this method. By including the contributions due to more and more bands, one can systematically arrive at the exact continuum solution from the lattice approximation, as we shall see. There is a large degrees of freedom in choosing the Block functions (1), as the original continuum theory has no crystal structure. These extra degrees of freedom are analogous to gauge fixing; the final answer to the continuum problem is independent of the particular choice of Bloch functions.

\section{SPURIOUS LATTICE FERMION SOLUTIONS}

To see the origin of the spurious lattice fermion solutions, we may consider the replacement of the continuum equation $-i \partial \psi / \partial x=p \psi$ by its discrete form in one space dimension:

$$
\frac{-i}{2 \ell}\left(\psi_{j+1}-\psi_{j-1}\right)=p_{L} \psi_{j}
$$

where $\psi_{j}$ is the value of $\psi$ at $j^{t h}$ site. The lattice-eigenvalue $p_{L}$ is given by

$$
p_{L}=\frac{1}{\ell} \sin K \ell
$$

where

$$
K \ell \equiv \theta
$$

is between $-\pi$ and $\pi$. The spurious solution refers to the zero $\left(p_{L}(\theta)=0\right)$ at $\theta=\pi$ (which is the same as $\theta=-\pi$ ). This is a special case of the NielsenNinomiya theorem [3].

\subsection{Elimination of Spurious Lattice Fermion Solutions}

We expand the continuum wave function $\psi(x)$ in terms of (1), in which the zeroth band $(n=0)$ is simply the linear interpolation of the discrete values $\left\{\psi_{j}\right\}$; i.e., in the zeroth-band approximation

$$
\psi(x)=\sum_{j} \psi_{j} \Delta(x-j \ell)
$$


where

$$
\Delta(x)= \begin{cases}1-\frac{|x|}{\ell} & \text { for }|x|<\ell \\ 0 & \text { otherwise. }\end{cases}
$$

Thus, at $x=j \ell, \psi(x)=\psi_{j}$. Substitute (5) into the continuum bilinear form

$$
B(\psi(x)) \equiv-i \int \psi(x)^{\dagger} \frac{d \psi(x)}{d x} d x
$$

Setting $\partial B / \partial \psi_{j}=0$ at a constant $\int \psi^{\dagger} \psi d x$, we find

$$
\psi_{j} \propto e^{i \theta j}
$$

with $\theta$ given by (4). Correspondingly, the zeroth-band Bloch function is

$$
f_{0}(K \mid x)=\sqrt{\frac{3}{N \ell(2+\cos \theta)}} \sum_{j} e^{i \theta j} \Delta(x-j \ell)
$$

where $N$ is the total number of lattice sites. It is not difficult to construct from $f_{0}(K \mid x)$ and the Fourier series a complete set of Bloch functions (1), which satisfy

$$
\int_{0}^{N \ell} f_{n}(K \mid x)^{*} f_{n^{\prime}}\left(K^{\prime} \mid x\right) d x=\delta_{n n^{\prime}} \delta_{K K^{\prime}}
$$

e.g., these can be given by, for $n \neq 0$,

$$
f_{n}(K \mid x) \equiv \frac{1}{\sqrt{N \ell}} e^{i K x+i 2 \pi n x / \ell}-\frac{a_{n}}{1+a_{0}}\left[f_{0}(K \mid x)+\frac{1}{\sqrt{N \ell}} e^{i K x}\right]
$$

where, for all $n$ including 0 ,

$$
a_{n}=\int_{0}^{N \ell} f_{0}(K \mid x)^{*} \frac{1}{\sqrt{N \ell}} e^{i K x+i 2 \pi n x / \ell}
$$

Let

$$
\beta_{n} \equiv-i \int f_{n}(K \mid x)^{*} \frac{\partial}{\partial x} f_{n}(K \mid x) d x
$$

We find for $n=0$

$$
\beta_{0}=\frac{3 \sin \theta}{2+\cos \theta}
$$

which, like (3), has a spurious zero solution at $\theta=\pi$.

If we substitute the full expansion

$$
\psi(x)=\sum_{n} \sum_{K} q_{n}(K) f_{n}(K \mid x)
$$

into (7), then $\delta B / \delta \psi(x)=0$ at a constant $\int \psi^{\dagger} \psi d x$ gives $-i \partial \psi / \partial x=p x$ where

$$
p=K+\frac{2 \pi m}{\ell}
$$




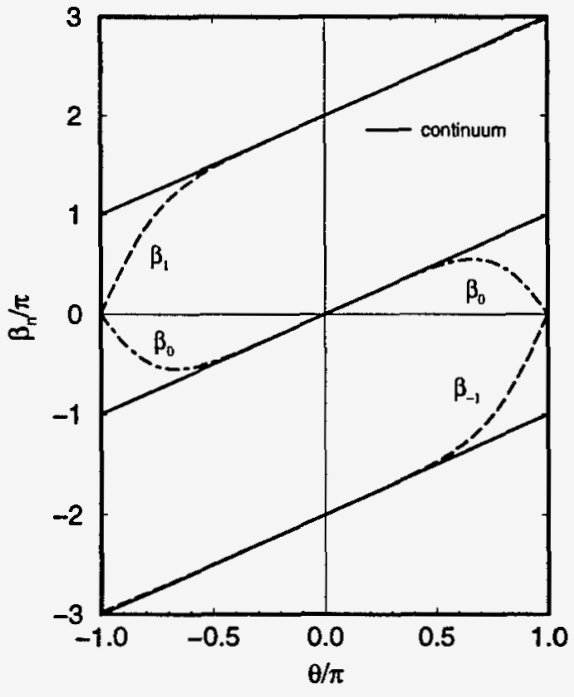

(a)

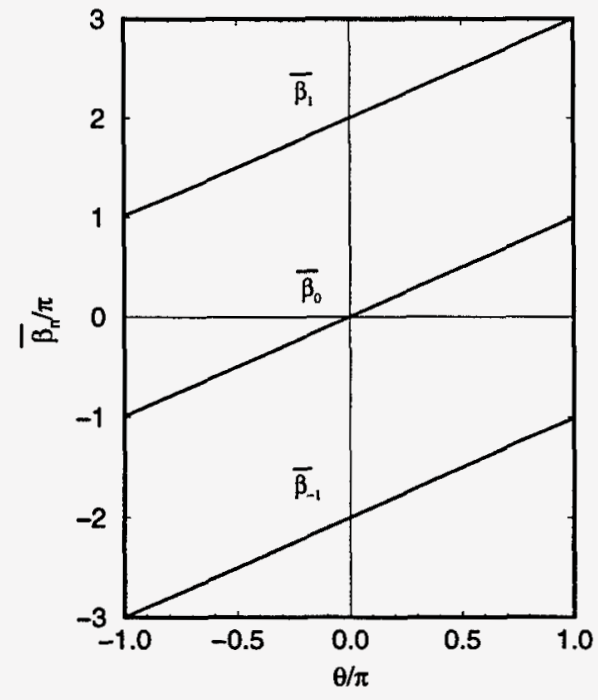

(b)

Fig. 1. - (a) The dash-dot line gives $\beta_{0}=3 \sin \theta /(2+\cos \ell)$ vs. $\theta=K \ell$ and the dashed lines denote $\beta_{-1}$ and $\beta_{1}$ defined by (13). The solid lines are the continuum free-particle spectrum $p \ell=K \ell+2 \pi m$ vs. $\theta$, with $m=0$ and \pm 1 . (b) Taking into account $n=0$ and $n= \pm 1$, we have removed all spurious zero-mode solutions.

with $K$ given by (4) and $m=\cdots,-1,0,1, \cdots$.

In Fig. 1, the abscissa is $\theta / \pi=K \ell / \pi$, the solid line gives the exact continuum value $p \ell / \pi$ and the different segments correspond to $m=-1,0,1$. The dashed line segments, in Fig. 1a are the corresponding $\beta_{n} / \pi$ defined by (13). For $|n|>1$, each $\beta_{n}$ deviates from the exact continuum result (16) within $<1 \%$. For $|n| \leq 1$, we see that $\beta_{0}$ and $\beta_{-1}$ are both 0 at $\theta=\pi$; likewise $\beta_{0}$ and $\beta_{1}$ are both 0 at $\theta=-\pi$. Thus, the spurious solutions also extend to $n= \pm 1$ bands. This additional unwanted degeneracy makes it easy to remove all spurious solutions, as we shall see.

Because $f_{0}(K \mid x)$ and $f_{1}(K \mid x)$ are not eigenfunctions of $-i \partial / \partial x$, the degeneracy between $\beta_{0}$ and $\beta_{-1}$ at $\theta=\pi$ can be removed by considering the off-diagonal elements of $-i \partial / \partial x$. At $\theta=\pi-\epsilon$ where $\epsilon$ is a positive infinitesimal, we may consider only two bands, $n=0$ and -1 ; in this subspace the operator $-i \partial / \partial x$ becomes the following $2 \times 2$ matrix:

$$
\left(\begin{array}{cc}
3 \epsilon & \sqrt{10} / \ell \\
\sqrt{10} / \ell & -5 \epsilon
\end{array}\right)
$$

As $\epsilon \rightarrow 0$, its eigenvalues are

$$
\pm \sqrt{10} / \ell= \pm 1.006 \times x / \ell .
$$


Similar considerations apply to $\theta=-\pi$ by taking into account the coupling between $n=0$ and $n=1$ bands. Thus, by taking into account only $n=0$ and \pm 1 , we have removed all spurious zero-mode solutions and, and in addition, the result differs from the exact continuum value by less than a few tenths of a percent for the entire range, see Fig. 1b.

\section{LUMP FUNCTIONS}

The function $\Delta(x)$, introduced in the zeroth-band approximation above is a special case of a general class of functions which we call lump functions. They are defined by

$$
L_{m}(x) \equiv \int_{-\infty}^{\infty} \frac{d k}{2 \pi} \frac{\left(2 \sin \frac{k}{2}\right)^{m}}{k^{m}} \times \begin{cases}e^{i k x / \ell} & \text { for } m \text { even } \\ e^{i k(x-\ell / 2) / \ell} & \text { for } m \text { odd }\end{cases}
$$

where $m$ is a positive integer $>0$ and $x$ is the continuous coordinate variable. We see that for $m$ even

$$
\ell \frac{d L_{m}(x)}{d x}=-L_{m-1}(x)+L_{m-1}(x+\ell)
$$

and for $m$ odd

$$
\ell \frac{d L_{m}(x)}{d x}=L_{m-1}(x)-L_{m-1}(x-\ell)
$$

Another useful property is, within the range $0<x<\ell$,

$$
\sum_{j=-(m-2) / 2}^{m / 2} L_{m}(x-j \ell)=\text { constant } \quad \text { for } m \text { even }
$$

and

$$
\sum_{j=-(m-1) / 2}^{(m-1) / 2} L_{m}(x-j \ell)=\text { constant } \quad \text { for } m \text { odd }
$$

which can be readily proven by noting that their derivatives are both zero, on account of (20) and (21). These type of functions are related to the mathematical problem called partition of one [4].

The lump functions of lower order, $m=1,2$ and 3, are particularly useful in our lattice formulations of quantum field theories. It is convenient to give these functions special names:

$$
\begin{gathered}
C(x) \equiv L_{1}(x)= \begin{cases}1 & \text { for } 0<x<\ell \\
0 & \text { otherwise }\end{cases} \\
\Delta(x) \equiv L_{2}(x)
\end{gathered}
$$


and

$S(x) \equiv L_{3}(x)= \begin{cases}\frac{1}{8}\left[\left(3-2\left|\frac{x}{\ell}-\frac{1}{2}\right|\right)^{2}-3\left(1-2\left|\frac{x}{\ell}-\frac{1}{2}\right|\right)^{2}\right] & \text { for }\left|x-\frac{\ell}{2}\right|<\frac{\ell}{2} \\ \frac{1}{8}\left[\left(3-2\left|\frac{x}{\ell}-\frac{1}{2}\right|\right)^{2}\right. & \text { for } \frac{\ell}{2}\left|x-\frac{\ell}{2}\right|<\frac{3 \ell}{2} \\ 0 & \text { otherwise. }\end{cases}$

\section{NONCOMPACT LATTICE QCD}

In order to extend the above consideration to QCD we have to construct an appropriate complete set of Bloch wavefunctions that is compatible to the gauge fixing condition. As we shall see, once that is done the restriction to the zeroth band $(n=0)$ gives a noncompact formulation [2] of lattice QCD. The exact continuum theory can be reached through inclusion of all $n=0$ and $n \neq 0$ bands, without requiring the lattice size $\ell \rightarrow 0$. This makes it possible, at a nonzero $\ell$, for the lattice coupling $g_{\ell}$ to act as the renormalized continuum coupling. All physical results in the continuum are, of course, independent of $\ell$.

To construct zeroth-band Bloch functions in the Coulomb gauge, we define

$$
\begin{gathered}
f(K \mid x) \equiv \sum_{j} e^{i K j l} \Delta(x-j \ell), \\
g(K \mid x) \equiv \sum_{j} e^{i K\left(j+\frac{1}{2}\right) l} S(x-j \ell),
\end{gathered}
$$

with $|K| \leq \pi / \ell$. Apart from a normalization constant, $f(K \mid x)$ is the same function given by (9). From (20), we see that

$$
\ell \frac{\partial}{\partial x} g(K \mid x)=2 i \sin \frac{\theta}{2} f(K \mid x)
$$

where $\theta \equiv K \ell$ and therefore $|\theta| \leq \pi$. Introduce

$$
\begin{aligned}
& F_{x}(\vec{K} \mid \vec{r})=c_{1} g\left(K_{1} \mid x\right) f\left(K_{2} \mid y\right) f\left(K_{3} \mid z\right) \\
& F_{y}(\vec{K} \mid \vec{r})=c_{2} f\left(K_{1} \mid x\right) g\left(K_{2} \mid y\right) f\left(K_{3} \mid z\right) \\
& F_{z}(\vec{K} \mid \vec{r})=c_{3} f\left(K_{1} \mid x\right) f\left(K_{2} \mid y\right) g\left(K_{3} \mid z\right)
\end{aligned}
$$

where $c_{1}, c_{2}$ and $c_{3}$ are normalization constants. On account of (29), the derivatives

$$
\frac{\partial F_{x}}{\partial x} \propto \frac{\partial F_{y}}{\partial y} \propto \frac{\partial F_{z}}{\partial z} \propto \prod_{a=1}^{3} f_{a}\left(K_{a} \mid x_{a}\right)
$$

For each given $\vec{K}$, it is straightforward to introduce two unit vectors $\vec{\epsilon}_{1}(\vec{K})$ and $\vec{\epsilon}_{2}(\vec{K})$, orthogonal to each other, and the vector function $\overrightarrow{\mathcal{F}}_{\tau}(\vec{K} \mid \vec{r})$ whose spatial components are

$$
\overrightarrow{\mathcal{F}}_{\tau}(\vec{K} \mid \vec{r})_{i}=\vec{\epsilon}_{\tau}(\vec{K})_{i} F_{i}(\vec{K} \mid \vec{r})
$$


so that

$$
\vec{\nabla} \cdot \overrightarrow{\mathcal{F}}_{\tau}(\vec{K} \mid \vec{r})=0
$$

at all continuum $\vec{r}$ for the polarization index $\tau=1$ or 2 . The gauge field operator, in the zeroth-band approximation, is

$$
\vec{A}^{m}(\vec{r}, t)=\sum_{\tau=1}^{2} \sum_{\vec{K}} q_{\tau}^{m}(\vec{K}, t) \overrightarrow{\mathcal{F}}_{\tau}(\vec{K} \mid \vec{r}) .
$$

The Coulomb gauge condition $\vec{\nabla} \cdot \vec{A}^{m}$ is satisfied everywhere. It is straightforward to generalize (11) for the construction of the higher-band Bloch functions by using Fourier series and the zeroth-band function given above.

In our formulation, the zeroth-band approximation is the noncompact lattice QCD, whose Hamiltonian $H$ has exactly the same continuum form derived by Christ and Lee [5] in Coulomb gauge, except for the replacement of the gauge field operators, its conjugate momenta, and the bare coupling constant in the continuum with the corresponding lattice operators and lattice coupling constant.

\section{Acknowledgments}

The authors wish to thank R. Friedberg and T. D. Lee for collaborating on this project. Y. P. acknowledges the support of Alfred P. Sloan Foundation. This project is also supported in part by U.S. Department of Energy under grants DE-FG02-92 ER40699, DOE-91ER40651, Task B, and DE-AC02-76CH00016.

\section{References}

[1] Friedberg R., Lee T. D., Pang Y., J. Math. Phys. 35 (1994) 5601.

[2] Friedberg R., Lee T. D., Pang Y., Ren H. C., Phys. Rev. D 52 (1995) 4053.

[3] Nielsen H. B., Ninomiya M. Nucl. Phys. B (185) 1981,20.

[4] Grange P., private communication.

[5] Christ N. H., Lee T. D., Phys. Rev. D 22 (1980) 939. 


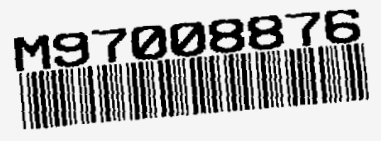

Report Number (14) BNL- $\frac{64611}{\text { CONE-970291 }=}$

Publ. Date (11)

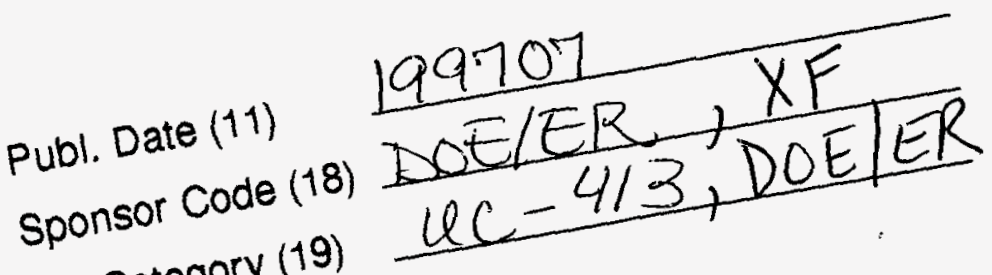
UC Category (19) UC -413 , 